KYUNGPOOK Math. J. 54(2014), 113-122

http://dx.doi.org/10.5666/KMJ.2014.54.1.113

\title{
Some Properties of $S$-metric Spaces and Fixed Point Results
}

\author{
ShaBAn SEDGHI \\ Department of Mathematics, Qaemshahr Branch, Islamic Azad University, Qaemshahr, \\ Iran \\ e-mail : sedghi_gh@yahoo.com \\ IshaK Altun* \\ Department of Mathematics, Faculty of Science and Arts, Kirikkale University, \\ 71450 Yahsihan, Kirikkale, Turkey \\ e-mail : ishakaltun@yahoo.com \\ NABI SHOBE \\ Department of Mathematics, Islamic Azad University-Babol Branch, Iran \\ e-mail: nabi_shobe@yahoo.com \\ Mohammad Ali Salahshour \\ Department of Mathematics, Islamic Azad University-Savadkooh Branch, Iran \\ e-mail : msalahshour2000@yahoo.com \\ ABstract. In this paper, we introduce $S$-metric spaces and give their some properties. \\ Also we present a common fixed point theorem for multivalued maps on complete $S$-metric \\ spaces. The single valued case and an illustrative example are given.
}

\section{Introduction}

In the present paper, we introduce the concept of $S$-metric spaces and give some properties of them. Then a common fixed point theorem for two multivalued mappings on complete $S$-metric spaces is given. In addition, we give an illustrative example for the single valued case.

We begin with the following definition.

Definition 1.1. Let $X$ be a nonempty set. An $S$-metric on $X$ is a function $S: X^{3} \rightarrow$ $[0, \infty)$ that satisfies the following conditions, for each $x, y, z, a \in X$,

1. $S(x, y, z) \geq 0$,

2. $S(x, y, z)=0$ if and only if $x=y=z$,

* Corresponding Author.

Received June 11, 2012; accepted May 5, 2013.

2010 Mathematics Subject Classification: 54H25, 47H10.

Key words and phrases: $S$-metric space, fixed point. 
3. $S(x, y, z) \leq S(x, x, a)+S(y, y, a)+S(z, z, a)$.

The pair $(X, S)$ is called an $S$-metric space.

Immediate examples of such $S$-metric spaces are:

1. Let $X=\mathbb{R}^{n}$ and $\|\cdot\|$ a norm on $X$, then $S(x, y, z)=\|y+z-2 x\|+\|y-z\|$ is an $S$-metric on $X$.

2. Let $X=\mathbb{R}^{n}$ and $\|\cdot\|$ a norm on $X$, then $S(x, y, z)=\|x-z\|+\|y-z\|$ is an $S$-metric on $X$.

3. Let $X$ be a nonempty set, $d$ is ordinary metric on $X$, then $S(x, y, z)=d(x, z)+$ $d(y, z)$ is an $S$-metric on $X$.

Lemma 1.2. In an $S$-metric space, we have $S(x, x, y)=S(y, y, x)$.

Proof. By third condition of $S$-metric, we have

$$
S(x, x, y) \leq S(x, x, x)+S(x, x, x)+S(y, y, x)=S(y, y, x)
$$

and similarly

$$
S(y, y, x) \leq S(y, y, y)+S(y, y, y)+S(x, x, y)=S(x, x, y) .
$$

Hence by (1.1) and (1.2), we get $S(x, x, y)=S(y, y, x)$.

Definition 1.3. Let $(X, S)$ be an $S$-metric space. For $r>0$ and $x \in X$ we define the open ball $B_{S}(x, r)$ and closed ball $B_{S}[x, r]$ with center $x$ and radius $r$ as follows respectively:

$$
\begin{gathered}
B_{S}(x, r)=\{y \in X: S(y, y, x)<r\}, \\
B_{S}[x, r]=\{y \in X: S(y, y, x) \leq r\} .
\end{gathered}
$$

Example 1.4. Let $X=\mathbb{R}$. Denote $S(x, y, z)=|y+z-2 x|+|y-z|$ for all $x, y, z \in \mathbb{R}$. Thus

$$
\begin{aligned}
B_{S}(1,2) & =\{y \in \mathbb{R}: S(y, y, 1)<2\}=\{y \in \mathbb{R}:|y-1|<1\} \\
& =\{y \in \mathbb{R}: 0<y<2\}=(0,2) .
\end{aligned}
$$

Definition 1.5. Let $(X, S)$ be an $S$-metric space and $A \subset X$.

1. If for every $x \in A$ there exists $r>0$ such that $B_{S}(x, r) \subset A$, then the subset $A$ is called open subset of $X$.

2. Subset $A$ of $X$ is said to be $S$-bounded if there exists $r>0$ such that $S(x, x, y)<r$ for all $x, y \in A$.

3. A sequence $\left\{x_{n}\right\}$ in $X$ converges to $x$ if and only if $S\left(x_{n}, x_{n}, x\right) \rightarrow 0$ as $n \rightarrow \infty$. That is for each $\varepsilon>0$ there exists $n_{0} \in \mathbb{N}$ such that

$$
\forall n \geq n_{0} \Longrightarrow S\left(x_{n}, x_{n}, x\right)<\varepsilon
$$

and we denote by $\lim _{n \rightarrow \infty} x_{n}=x$. 
4. Sequence $\left\{x_{n}\right\}$ in $X$ is called a Cauchy sequence if for each $\varepsilon>0$, there exists $n_{0} \in \mathbb{N}$ such that $S\left(x_{n}, x_{n}, x_{m}\right)<\varepsilon$ for each $n, m \geq n_{0}$.

5. The $S$-metric space $(X, S)$ is said to be complete if every Cauchy sequence is convergent.

6. Let $\tau$ be the set of all $A \subset X$ with $x \in A$ if and only if there exists $r>0$ such that $B_{S}(x, r) \subset A$. Then $\tau$ is a topology on $X$ (induced by the $S$-metric $S$ ).

Lemma 1.6. Let $(X, S)$ be an $S$-metric space. If $r>0$ and $x \in X$, then the ball $B_{S}(x, r)$ is open subset of $X$.

Proof. Let $y \in B_{S}(x, r)$, hence $S(y, y, x)<r$. If set $\delta=S(x, x, y)$ and $r^{\prime}=\frac{r-\delta}{2}$ then we prove that $B_{S}\left(y, r^{\prime}\right) \subseteq B_{S}(x, r)$. Let $z \in B_{S}\left(y, r^{\prime}\right)$, then $S(z, z, y)<r^{\prime}$. By third condition of $S$-metric we have

$$
S(z, z, x) \leq S(z, z, y)+S(z, z, y)+S(x, x, y)<2 r^{\prime}+\delta=r
$$

Hence $B_{S}\left(y, r^{\prime}\right) \subseteq B_{S}(x, r)$. That is the ball $B_{S}(x, r)$ is a open subset of $X$.

Lemma 1.7. Let $(X, S)$ be an $S$-metric space. If sequence $\left\{x_{n}\right\}$ in $X$ converges to $x$, then $x$ is unique.

Proof. Let $\left\{x_{n}\right\}$ converges to $x$ and $y$, then for each $\varepsilon>0$ there exist $n_{1}, n_{2} \in \mathbb{N}$ such that

$$
\forall n \geq n_{1} \Longrightarrow S\left(x_{n}, x_{n}, x\right)<\frac{\varepsilon}{4}
$$

and

$$
\forall n \geq n_{2} \Longrightarrow S\left(x_{n}, x_{n}, y\right)<\frac{\varepsilon}{2} .
$$

If set $n_{0}=\max \left\{n_{1}, n_{2}\right\}$, then for every $n \geq n_{0}$ by third condition $S$-metric we have:

$$
S(x, x, y) \leq 2 S\left(x, x, x_{n}\right)+S\left(y, y, x_{n}\right)<\frac{\varepsilon}{2}+\frac{\varepsilon}{2}=\varepsilon .
$$

Hence $S(x, x, y)=0$ so $x=y$.

Lemma 1.8. Let $(X, S)$ be an $S$-metric space. If sequence $\left\{x_{n}\right\}$ in $X$ is converges to $x$, then $\left\{x_{n}\right\}$ is a Cauchy sequence.

Proof. Since $\lim _{n \rightarrow \infty} x_{n}=x$ then for each $\varepsilon>0$ there exists $n_{1}, n_{2} \in \mathbb{N}$ such that

$$
n \geq n_{1} \Rightarrow S\left(x_{n}, x_{n}, x\right)<\frac{\varepsilon}{4}
$$

and

$$
m \geq n_{2} \Rightarrow S\left(x_{m}, x_{m}, x\right)<\frac{\varepsilon}{2} .
$$

If set $n_{0}=\max \left\{n_{1}, n_{2}\right\}$, then for every $n, m \geq n_{0}$ by third condition of $S$-metric we have:

$$
S\left(x_{n}, x_{n}, x_{m}\right) \leq 2 S\left(x_{n}, x_{n}, x\right)+S\left(x_{m}, x_{m}, x\right)<\frac{\varepsilon}{2}+\frac{\varepsilon}{2}=\varepsilon .
$$

Hence $\left\{x_{n}\right\}$ is a Cauchy sequence.

Lemma 1.9. Let $(X, S)$ be an $S$ - metric space. If there exist sequences $\left\{x_{n}\right\}$ and $\left\{y_{n}\right\}$ such that $\lim _{n \rightarrow \infty} x_{n}=x$ and $\lim _{n \rightarrow \infty} y_{n}=y$, then

$$
\lim _{n \rightarrow \infty} S\left(x_{n}, x_{n}, y_{n}\right)=S(x, x, y) .
$$


Proof. Since $\lim _{n \rightarrow \infty} x_{n}=x$ and $\lim _{n \rightarrow \infty} y_{n}=y$, then for each $\varepsilon>0$ there exist $n_{1}, n_{2} \in \mathbb{N}$ such that

$$
\forall n \geq n_{1} \Rightarrow S\left(x_{n}, x_{n}, x\right)<\frac{\varepsilon}{4}
$$

and

$$
\forall n \geq n_{2} \Rightarrow S\left(y_{n}, y_{n}, y\right)<\frac{\varepsilon}{4} .
$$

If set $n_{0}=\max \left\{n_{1}, n_{2}\right\}$, then for every $n \geq n_{0}$ by third condition of $S$-metric we have:

$$
\begin{aligned}
S\left(x_{n}, x_{n}, y_{n}\right) & \leq 2 S\left(x_{n}, x_{n}, x\right)+S\left(y_{n}, y_{n}, x\right) \\
& \leq 2 S\left(x_{n}, x_{n}, x\right)+2 S\left(y_{n}, y_{n}, y\right)+S(x, x, y) \\
& <\frac{\varepsilon}{2}+\frac{\varepsilon}{2}+S(x, x, y)=\varepsilon+S(x, x, y) .
\end{aligned}
$$

Hence we have:

$$
S\left(x_{n}, x_{n}, y_{n}\right)-S(x, x, y)<\varepsilon .
$$

On the other hand, we have

$$
\begin{aligned}
S(x, x, y) & \leq 2 S\left(x, x, x_{n}\right)+S\left(y, y, x_{n}\right) \\
& \leq 2 S\left(x, x, x_{n}\right)+2 S\left(y, y, y_{n}\right)+S\left(x_{n}, x_{n}, y_{n}\right) \\
& <\frac{\varepsilon}{2}+\frac{\varepsilon}{2}+S\left(x_{n}, x_{n}, y_{n}\right)=\varepsilon+S\left(x_{n}, x_{n}, y_{n}\right)
\end{aligned}
$$

that is

$$
S(x, x, y)-S\left(x_{n}, x_{n}, y_{n}\right)<\varepsilon .
$$

Therefore by relations (1.3) and (1.4) we have $\left|S\left(x_{n}, x_{n}, y_{n}\right)-S(x, x, y)\right|<\varepsilon$, that is

$$
\lim _{n \rightarrow \infty} S\left(x_{n}, x_{n}, y_{n}\right)=S(x, x, y) .
$$

Let $(X, S)$ be an $S$-metric space, $C(X)$ denotes the family of all nonempty closed subsets of $X$. For $A$ and $B$ two nonempty subsets of $X$ we define;

$$
\operatorname{dist}(x, A)=\inf _{a \in A}\{S(x, x, a)\}
$$

and

$$
\mathcal{S}(A, A, B)=\sup _{a \in A, b \in B}\{S(a, a, b)\} .
$$

By the definition of $\operatorname{dist}(x, A)$, it is clear that $\operatorname{dist}(x, A)=0 \Leftrightarrow x \in \bar{A}$.

\section{Implicit Relations}

Implicit relations on metric spaces have been used in many articles. For examples, [1], [2], [3], [4], [5], [6], [7], [8]. Let $\mathbb{R}_{+}$be the set of nonnegative real numbers and let $\mathcal{T}$ be the set of all functions $T: \mathbb{R}_{+}^{6} \rightarrow \mathbb{R}$ satisfying the following conditions: 
$T_{0}: T\left(\liminf _{n \rightarrow \infty} p_{n}\right) \leq \liminf _{n \rightarrow \infty} T\left(p_{n}\right)$ for any $p_{n} \in \mathbb{R}_{+}^{6}$, where $\liminf _{n \rightarrow \infty} p_{n}$ means component-wise liminf.

$T_{1}: T\left(t_{1}, \ldots, t_{6}\right)$ is nonincreasing in $t_{2}, \ldots, t_{6}$.

$T_{2}$ : there exists a continuous strictly increasing function $\phi: \mathbb{R}_{+} \rightarrow \mathbb{R}_{+}$with $\phi(t)<t$ for $t>0$ and $\varepsilon>0$ such that the inequalities

$$
u \leq w+\varepsilon
$$

and

$$
T(w, v, v, u, 2 u+v, 0) \leq 0 \quad \text { or } \quad T(w, v, u, v, 0,2 u+v) \leq 0
$$

implies $w \leq \phi(v)$.

$T_{3}: T(w, 0, v, 0,0, v) \leq 0$ and $T(w, 0,0, v, v, 0) \leq 0$ implies $w \leq \phi(v)$, where $\phi$ is the function in $T_{2}$.

Example 2.1. $T\left(t_{1}, \ldots, t_{6}\right)=t_{1}-f\left(\max \left\{t_{2}, t_{3}, t_{4}, \frac{1}{3}\left(t_{5}+t_{6}\right)\right\}\right)$, where $f: \mathbb{R}_{+} \rightarrow \mathbb{R}_{+}$ continuous strictly increasing function with $f(t)<t$ for $t>0$.

$T_{0}$ and $T_{1}$ : Obviously.

$T_{2}$ : Let $u>0$, then choose $\varepsilon>0$ so that $f(u)+\varepsilon<u$ (this is possible since $f(u)<u$ ). Now let $u \leq w+\varepsilon$ and $T(w, v, v, u, 2 u+v, 0)=w-f(\max \{u, v\}) \leq 0$. If $u \geq v$, then $u \leq w+\varepsilon \leq f(u)+\varepsilon<u$, a contradiction. Thus $u<v$ and $w \leq f(v)$. Similarly, $u \leq w+\varepsilon$ and $T(w, v, u, v, 0,2 u+v) \leq 0$ imply $w \leq f(v)$. If $u=0$, then $w \leq f(v)$. Thus $T_{2}$ is satisfied with $\phi=f$.

$T_{3}: T(w, 0, v, 0,0, v)=T(w, 0,0, v, v, 0)=w-f(v) \leq 0 \Rightarrow w \leq f(v)=\phi(v)$.

\section{Fixed Point Theory}

Our main result for fixed point theory of this work as follows.

Theorem 3.1. Let $(X, S)$ be a complete $S$-metric space, $x_{0} \in X, r>0$ with $F, G$ : $B_{S}\left[x_{0}, r\right] \rightarrow C(X)$. Suppose, for all $x, y \in B_{S}\left[x_{0}, r\right]$ sets $F x, G y$ are bounded and

(3.1) $T(\mathcal{S}(F x, F x, G y), S(x, x, y), \operatorname{dist}(x, F x), \operatorname{dist}(y, G y), \operatorname{dist}(x, G y), \operatorname{dist}(y, F x)) \leq 0$

where $T \in \mathcal{T}$. Also assume the following conditions are satisfied:

$$
\operatorname{dist}\left(x_{0}, F x_{0}\right)<\frac{r-\phi(r)}{2}
$$

and

$$
\sum_{i=1}^{\infty} \phi^{i}\left(\frac{r-\phi(r)}{2}\right) \leq \frac{\phi(r)}{2}
$$

where $\phi$ is the function in $T_{2}$. Then there exists $x \in B_{S}\left[x_{0}, r\right]$ with $x \in F x$ and $x \in G x$.

Proof. From (3.2) we can choose $x_{1} \in F x_{0}$ with

$$
S\left(x_{0}, x_{0}, x_{1}\right)<\frac{r-\phi(r)}{2}
$$


Hence $S\left(x_{1}, x_{1}, x_{0}\right)<r$ so $x_{1} \in B_{S}\left[x_{0}, r\right]$. Since $\phi$ is strictly increasing by (3.4) we can choose $\varepsilon>0$ such that

$$
\phi\left(S\left(x_{0}, x_{0}, x_{1}\right)\right)+\varepsilon<\phi\left(\frac{r-\phi(r)}{2}\right) .
$$

On the other hand, for this $\varepsilon$ there is $x_{2} \in G x_{1}$ so that

$$
S\left(x_{1}, x_{1}, x_{2}\right) \leq \operatorname{dist}\left(x_{1}, G x_{1}\right)+\varepsilon \leq \mathcal{S}\left(F x_{0}, F x_{0}, G x_{1}\right)+\varepsilon .
$$

Now since $x_{0}, x_{1} \in B_{S}\left[x_{0}, r\right]$ we can use the inequality (3.1) to obtain

$$
\begin{gathered}
T\left(\mathcal{S}\left(F x_{0}, F x_{0}, G x_{1}\right), S\left(x_{0}, x_{0}, x_{1}\right), \operatorname{dist}\left(x_{0}, F x_{0}\right), \operatorname{dist}\left(x_{1}, G x_{1}\right),\right. \\
\left.\operatorname{dist}\left(x_{0}, G x_{1}\right), \operatorname{dist}\left(x_{1}, F x_{0}\right)\right) \leq 0 .
\end{gathered}
$$

From $T_{1}$ we have

$$
T\left(\mathcal{S}\left(F x_{0}, F x_{0}, G x_{1}\right), S\left(x_{0}, x_{0}, x_{1}\right), S\left(x_{0}, x_{0}, x_{1}\right), S\left(x_{1}, x_{1}, x_{2}\right), S\left(x_{0}, x_{0}, x_{2}\right), 0\right) \leq 0,
$$

that is

$$
T(w, v, v, u, 2 u+v, 0) \leq 0,
$$

where $w=\mathcal{S}\left(F x_{0}, F x_{0}, G x_{1}\right), v=S\left(x_{0}, x_{0}, x_{1}\right)$ and $u=S\left(x_{1}, x_{1}, x_{2}\right)$. Therefore, from $T_{2}$,

and (3.6) yields

$$
\mathcal{S}\left(F x_{0}, F x_{0}, G x_{1}\right) \leq \phi\left(S\left(x_{0}, x_{0}, x_{1}\right)\right)
$$

$$
S\left(x_{1}, x_{1}, x_{2}\right) \leq \phi\left(S\left(x_{0}, x_{0}, x_{1}\right)\right)+\varepsilon .
$$

Thus from (3.5) we have:

$$
S\left(x_{1}, x_{1}, x_{2}\right)<\phi\left(\frac{r-\phi(r)}{2}\right)
$$

Now by (3.3), (3.4), (3.7) and third condition of $S$-metric have:

$$
\begin{aligned}
S\left(x_{2}, x_{2}, x_{0}\right)=S\left(x_{0}, x_{0}, x_{2}\right) & \leq 2 S\left(x_{0}, x_{0}, x_{1}\right)+S\left(x_{1}, x_{1}, x_{2}\right) \\
& <r-\phi(r)+\phi\left(\frac{r-\phi(r)}{2}\right) \\
& <r-\phi(r)+2 \sum_{i=1}^{\infty} \phi^{i}\left(\frac{r-\phi(r)}{2}\right) \leq r
\end{aligned}
$$

so $x_{2} \in B_{S}\left[x_{0}, r\right]$. Again by (3.7) and strictly increasing $\phi$ there is $\delta>0$ so that

$$
\phi\left(S\left(x_{1}, x_{1}, x_{2}\right)\right)+\delta<\phi^{2}\left(\frac{r-\phi(r)}{2}\right),
$$

also for this $\delta>0$ there is $x_{3} \in F x_{2}$ so that

$$
S\left(x_{2}, x_{2}, x_{3}\right) \leq \operatorname{dist}\left(x_{2}, F x_{2}\right)+\delta \leq \mathcal{S}\left(G x_{1}, G x_{1}, F x_{2}\right)+\delta .
$$

As above, since $x_{1}, x_{2} \in B_{S}\left[x_{0}, r\right]$ we can use the inequality (3.1) to obtain

$$
T\left(\mathcal{S}\left(F x_{2}, F x_{2}, G x_{1}\right), S\left(x_{2}, x_{2}, x_{1}\right), \operatorname{dist}\left(x_{2}, F x_{2}\right),\right.
$$




$$
\left.\operatorname{dist}\left(x_{1}, G x_{1}\right), \operatorname{dist}\left(x_{2}, G x_{1}\right), \operatorname{dist}\left(x_{1}, F x_{2}\right)\right) \leq 0
$$

and so from $T_{1}$ we have

$$
T\left(\mathcal{S}\left(F x_{2}, F x_{2}, G x_{1}\right), S\left(x_{2}, x_{2}, x_{1}\right), S\left(x_{2}, x_{2}, x_{3}\right), S\left(x_{1}, x_{1}, x_{2}\right), 0, S\left(x_{1}, x_{1}, x_{3}\right)\right) \leq 0
$$

that is

$$
T(w, v, u, v, 0,2 u+v) \leq 0,
$$

where $w=\mathcal{S}\left(F x_{2}, F x_{2}, G x_{1}\right), v=S\left(x_{1}, x_{1}, x_{2}\right)$ and $u=S\left(x_{2}, x_{2}, x_{3}\right)$. Therefore from $T_{2}$,

$$
w \leq \phi(v)
$$

that is

and so (3.9) gives

$$
\mathcal{S}\left(F x_{2}, F x_{2}, G x_{1}\right) \leq \phi\left(S\left(x_{1}, x_{1}, x_{2}\right)\right)
$$

$$
S\left(x_{2}, x_{2}, x_{3}\right) \leq \phi\left(S\left(x_{1}, x_{1}, x_{2}\right)\right)+\delta .
$$

Thus from (3.8) we have

$$
S\left(x_{2}, x_{2}, x_{3}\right)<\phi^{2}\left(\frac{r-\phi(r)}{2}\right) .
$$

Now (3.3), (3.4), (3.7), (3.10) and third condition of $S$-metric implies:

$$
\begin{aligned}
S\left(x_{3}, x_{3}, x_{0}\right)=S\left(x_{0}, x_{0}, x_{3}\right) & \leq 2 S\left(x_{0}, x_{0}, x_{1}\right)+2 S\left(x_{1}, x_{1}, x_{2}\right)+S\left(x_{2}, x_{2}, x_{3}\right) \\
& <r-\phi(r)+2 \phi\left(\frac{r-\phi(r)}{2}\right)+\phi^{2}\left(\frac{r-\phi(r)}{2}\right) \\
& \leq r-\phi(r)+2 \sum_{i=1}^{\infty} \phi^{i}\left(\frac{r-\phi(r)}{2}\right) \leq r
\end{aligned}
$$

Thus $x_{3} \in B_{S}\left[x_{0}, r\right]$.

Continuing this way we can obtain a sequence $\left\{x_{n}\right\} \subseteq B_{S}\left[x_{0}, r\right]$ such that $x_{2 n+2} \in$ $G x_{2 n+1}$ and $x_{2 n+1} \in F x_{2 n}$ for $n \geq 0$ and

$$
S\left(x_{n}, x_{n}, x_{n+1}\right)<\phi^{n}\left(\frac{r-\phi(r)}{2}\right) .
$$

Next we show that $\left\{x_{n}\right\}$ is a Cauchy sequence. Notice by (3.3) and above inequality for each $n, m \in \mathbb{N}$ with $m>n$ we have:

$$
\begin{aligned}
S\left(x_{n}, x_{n}, x_{m}\right) & \leq 2 \sum_{i=n}^{m-2} S\left(x_{i}, x_{i}, x_{i+1}\right)+S\left(x_{m-1}, x_{m-1}, x_{m}\right) \\
& \leq 2 \sum_{i=n}^{m-1} S\left(x_{i}, x_{i}, x_{i+1}\right)<2 \sum_{i=n}^{m-1} \phi^{i}\left(\frac{r-\phi(r)}{2}\right) \\
& \leq 2 \sum_{i=n}^{\infty} \phi^{i}\left(\frac{r-\phi(r)}{2}\right)
\end{aligned}
$$

so (3.3) guarantees that $\left\{x_{n}\right\}$ is a Cauchy sequence. Thus there exists $x \in B_{S}\left[x_{0}, r\right]$ with $x_{n} \rightarrow x$. It remains to show $x \in F x$ and $x \in G x$. For $n$ even (since $x_{n}, x \in B_{S}\left[x_{0}, r\right]$ ) we can use the inequality (3.1), we have

$$
T\left(\mathcal{S}\left(F x, F x, G x_{n-1}\right), S\left(x, x, x_{n-1}\right), \operatorname{dist}(x, F x), \operatorname{dist}\left(x_{n-1}, G x_{n-1}\right),\right.
$$




$$
\left.\operatorname{dist}\left(x, G x_{n-1}\right), \operatorname{dist}\left(x_{n-1}, F x\right)\right) \leq 0 .
$$

Now taking limit inferior as $n \rightarrow \infty$ (using $\left.T_{0}\right)$ we have (notice $\operatorname{dist}\left(x, G x_{n-1}\right) \leq$ $S\left(x, x, x_{n}\right) \rightarrow 0$, and also $\left.\operatorname{dist}\left(x_{n-1}, G x_{n-1}\right) \leq S\left(x_{n-1}, x_{n-1}, x_{n}\right) \rightarrow 0\right)$

$$
T\left(\operatorname{liminfS}_{n \rightarrow \infty}\left(F x, F x, G x_{n-1}\right), 0, \operatorname{dist}(x, F x), 0,0, \operatorname{dist}(x, F x)\right) \leq 0 .
$$

From $T_{3}$ we have

$$
\liminf _{n \rightarrow \infty} \mathcal{S}\left(F x, F x, G x_{n-1}\right) \leq \phi(\operatorname{dist}(x, F x))
$$

Now

$$
\operatorname{dist}(x, F x) \leq 2 S\left(x, x, x_{n}\right)+\operatorname{dist}\left(x_{n}, F x\right) \leq 2 S\left(x, x, x_{n}\right)+\mathcal{S}\left(G x_{n-1}, G x_{n-1}, F x\right)
$$

and so

$$
\operatorname{dist}(x, F x) \leq 0+\liminf _{n \rightarrow \infty} \mathcal{S}\left(F x, F x, G x_{n-1}\right) \leq \phi(\operatorname{dist}(x, F x)) .
$$

Thus $\operatorname{dist}(x, F x)=0$ since $\phi(t)<t$ for $t>0$, so $x \in \overline{F x}=F x$.

For $n$ odd ,

$$
\operatorname{dist}(x, G x) \leq S\left(x, x, x_{n}\right)+\operatorname{dist}\left(x_{n}, G x\right) \leq S\left(x, x, x_{n}\right)+\mathcal{S}\left(F x_{n-1}, F x_{n-1}, G x\right),
$$

and as above we obtain $\operatorname{dist}(x, G x)=0$, so $x \in G x$.

Now we give some corollaries.

Corollary 3.2. Let $(X, S)$ be a complete $S$-metric space, $x_{0} \in X, r>0$ with $F, G$ : $B_{S}\left[x_{0}, r\right] \rightarrow C(X)$. Suppose, for all $x, y \in B_{S}\left[x_{0}, r\right]$ sets $F x, G y$ are bounded and

$$
\mathcal{S}(F x, F x, G y) \leq k \max \left\{S(x, x, y), \operatorname{dist}(x, F x), \operatorname{dist}(y, G y), \frac{\operatorname{dist}(x, G y)}{3}, \frac{\operatorname{dist}(y, F x)}{3}\right\}
$$

where $0<k<1$. Also assume the following condition is satisfied:

$$
\operatorname{dist}\left(x_{0}, F x_{0}\right)<\frac{1-k}{2} r .
$$

Then there exists $x \in B_{S}\left[x_{0}, r\right]$ with $x \in F x$ and $x \in G x$.

Proof. By Theorem 3.1 , it is enough to set $T\left(t_{1}, t_{2}, \ldots, t_{6}\right)=t_{1}-k \max \left\{t_{2}, t_{3}, t_{4}, \frac{t_{5}}{3}, \frac{t_{6}}{3}\right\}$. In this case, $\phi(t)=k t$ and

$$
\sum_{i=1}^{\infty} \phi^{i}\left(\frac{r-\phi(r)}{2}\right)=\frac{k r}{2}=\frac{\phi(r)}{2} .
$$

Corollary 3.3. Let $(X, S)$ be a complete $S$-metric space, $x_{0} \in X, r>0$ with $F, G$ : $B_{S}\left[x_{0}, r\right] \rightarrow X$. Suppose for all $x, y \in B_{S}\left[x_{0}, r\right]$,

$$
S(F x, F x, G y) \leq k \max \left\{S(x, x, y), S(x, x, F x), S(y, y, G y), \frac{S(x, x, G y)}{3}, \frac{S(y, y, F x)}{3}\right\}
$$

where $0<k<1$. Also assume the following condition is satisfied:

$$
S\left(x_{0}, x_{0}, F x_{0}\right)<\frac{1-k}{2} r .
$$


Then there exists a unique $x \in B_{S}\left[x_{0}, r\right]$ with $F x=G x=x$.

Proof. By Corollary 3.2, there exists an $x \in X$ such that $F x=G x=x$. It is enough prove that $x$ is unique.

Let $y$ be another common fixed point of $F$ and $G$, that is $y=F y=G y$, then we have

$$
\begin{aligned}
S(x, x, y)=S(F x, F x, G y) & \leq k \max \{S(x, x, y), S(x, x, x), S(y, y, y)\} \\
& =k S(x, x, y),
\end{aligned}
$$

which is a contradiction. Therefore $F$ and $G$ have a unique common fixed point in $B_{S}\left[x_{0}, r\right]$.

Corollary 3.4. Let $(X, S)$ be a complete $S$-metric space, $x_{0} \in X, r>0$ with $F$ : $B_{S}\left[x_{0}, r\right] \rightarrow X$. Suppose for all $x, y \in B_{S}\left[x_{0}, r\right]$,

$$
S(F x, F x, F y) \leq k \max \left\{S(x, x, y), S(x, x, F x), S(y, y, F y), \frac{S(x, x, F y)}{3}, \frac{S(y, y, F x)}{3}\right\}
$$

where $0<k<1$. Also assume the following condition is satisfied:

$$
S\left(x_{0}, x_{0}, F x_{0}\right)<\frac{1-k}{2} r .
$$

Then there exists a unique $x \in B_{S}\left[x_{0}, r\right]$ with $F x=x$.

Now we give an example.

Example 3.5. Let $X=\mathbb{R}$ and $S(x, y, z)=|x-z|+|y-z|$. Then $(X, S)$ is a complete $S$-metric space. Let $x_{0}=1$ and $r=6$, then

$$
\begin{aligned}
B_{S}\left[x_{0}, r\right] & =B_{S}[1,6] \\
& =\{y \in X: S(y, y, x) \leq 6\} \\
& =[-2,4] .
\end{aligned}
$$

Now let $F: B_{S}\left[x_{0}, r\right] \rightarrow X, F x=\frac{x}{2}$ and let $k=\frac{1}{2}$, then

$$
S\left(x_{0}, x_{0}, F x_{0}\right)=S\left(1,1, \frac{1}{2}\right)=1<\frac{3}{2}=\frac{1-k}{2} r .
$$

Also, for all $x, y \in B_{S}\left[x_{0}, r\right]$, we have

$$
\begin{aligned}
S(F x, F x, F y) & =2|F x-F y| \\
& =|x-y| \\
& =\frac{1}{2}(2|x-y|) \\
& =\frac{1}{2} S(x, x, y) \\
& \leq \frac{1}{2} \max \left\{S(x, x, y), S(x, x, F x), S(y, y, F y), \frac{S(x, x, F y)}{3}, \frac{S(y, y, F x)}{3}\right\} .
\end{aligned}
$$

Therefore all conditions of Corollary 3.4 are satisfied, thus $F$ has a unique fixed point in $B_{S}\left[x_{0}, r\right]=[-2,4]$. 


\section{References}

[1] I. Altun, Fixed points and homotopy results for multivalued mappings satisfying an implicit relation, J. Fixed Point Theory and Appl., 9(2011), 125-134.

[2] I. Altun and D. Turkoglu, Some fixed point theorems for weakly compatible mappings satisfying an implicit relation, Taiwanese J. Math., 13(4)(2009), 1291-1304.

[3] I. Beg, A. R. Butt, Fixed point for set-valued mappings satisfying an implicit relation in partially ordered metric spaces, Nonlinear Anal., 71(9)(2009), 3699-3704.

[4] M. Imdad, S. Kumar and M. S. Khan, Remarks on some fixed point theorems satisfying implicit relations, Rad. Math., 11(1)(2002), 135-143.

[5] V. Popa, A general coincidence theorem for compatible multivalued mappings satisfying an implicit relation, Demonstratio Math., 33(1)(2000), 159-164.

[6] V. Popa, Some fixed point theorems for compatible mappings satisfying an implicit relation, Demonstratio Math., 32(1)(1999), 157-163.

[7] S. Sedghi, I. Altun and N. Shobe, A fixed point theorem for multi-maps satisfying an implicit relation on metric spaces, Appl. Anal. Discrete Math., 2(2)(2008), 189-196.

[8] S. Sharma and B. Desphande, On compatible mappings satisfying an implicit relation in common fixed point consideration, Tamkang J. Math., 33(3)(2002), 245-252. 